\title{
TSR@ISOLDE - The First Storage Ring Facility at an ISOL Facility
}

\author{
Fredrik Wenander for the TSR@ISOLDE working group \\ BE Department \\ 1211-Geneva-23, Switzerland \\ E-mail: fredrik.wenander@cern.ch
}

It has been proposed to use the Test Storage Ring (TSR) from MPI-K, Heidelberg [1,2], at the High Intensity Energy Isolde (HIE-ISOLDE) facility to perform experiments with stored exotic nuclides. With the integration of TSR at HIE-ISOLDE it would become the first storage ring at an isotope separator on-line (ISOL) facility. This, in combination with the capability of going to low beam energies (some $\mathrm{MeV} / \mathrm{u}$ ), opens up a broad area of research and offers unique opportunities for studies in nuclear structure and nuclear astrophysics. A substantial fraction of the vast number of radioactive beams produced at the ISOLDE facility could be injected into the ring for storage, beam deceleration or acceleration. As injector, the superconducting HIEISOLDE Linac could be used.

The project layout at its present stage will be summarized, including the interfacing of the ring with the HIE-ISOLDE Linac beam lines. The different operational possibilities and constraints that arise when the ring is connected to an ISOL facility with a successive charge breeder will be addressed. The aspects of beam storage life-time, with and without an internal gas jet target, and attainable beam intensities at these energies will be discussed. The possibilities to perform beam cleaning inside the ring to suppress isobaric contaminants which can be present from the ISOL production stage will be evaluated. Finally, the ion charge-state requirements will be mentioned among other items.

This paper only lists some of the issues that have been addressed. A more in-depth description of the project, including a survey of the possible physics cases, is given in a Technical Design Report [3].

8th International Conference on Nuclear Physics at Storage Rings (Stori11)

Laboratori Nazionali di Frascati dell'INFN , Italy

October 9-14, 2011 


\section{Motivation}

By combining an ISOL facility with a storage ring, higher intensities and cooler beams could be achievable when compared to an equivalent in-flight and storage ring combination. Compared to a thick experimental target (with a thickness of a approximately $10^{19}$ atoms $/ \mathrm{cm}^{2}$ ) placed directly after the post-accelerated radioactive beam with a single beam passage, a thin inring target (some $10^{12}$ to $10^{13}$ atoms $/ \mathrm{cm}^{2}$ ) with a re-circulating beam will lead to: less background radiation from the target container and beam dump; reduced energy straggling for projectile and reaction products; smaller beam size yielding improved kinematic corrections for reaction products; and decreased detector dead-time compared with the operation at REXISOLDE where the beam is pulsed (which has a duty factor $5 \cdot 10^{-4}$ to $5 \cdot 10^{-3}$ ).

\section{Present status of TSR and ISOLDE}

The REX-ISOLDE post-accelerator at CERN [4] is capable of accelerating most of the over 800 isotopes produced at ISOLDE, comprising of 70 elements. Presently the beam energy is limited to approximately $3 \mathrm{MeV} / \mathrm{u}$, but with an on-going upgrade energies of $5 \mathrm{MeV} / \mathrm{u}$, and later of at least $10 \mathrm{MeV} / \mathrm{u}$, are to be available for ion mass-to-charge ratios less than 4.5 [5].

The TSR at Heidelberg is providing stable beams for mainly atomic physics studies and accelerator development. The ion injection is done from a $12 \mathrm{MV}$ MP-tandem or a high current injector, with a possibility to boost the beam energy in a post accelerator of similar type as REX-ISOLDE. The $55.4 \mathrm{~m}$ circumference ring is equipped with an electron cooler. In the one of the straight sections of the ring a dedicated ultracold electron target can be inserted or a gas jet target.

\section{Technical aspects of connecting TSR with ISOLDE}

\subsection{Beam energy}

The radioactive ions in the ring can either be circulating with the injection energy or be further accelerated or decelerated. The rigidity limits of the storage ring and the mass-to-charge range available with REX-ISOLDE set the boundaries. In principle beam energies between 0.5 and $>25 \mathrm{MeV} / \mathrm{u}$ are attainable for the appropriate mass-to-charge ratio.

\subsection{Electron cooling}

Several of the proposed experiments either require, or would benefit from, an electroncooled ion beam as the beam diameter in the target position would be reduced from $\sim 36 \mathrm{~mm}$ to $\sim 1 \mathrm{~mm}$ and the longitudinal momentum spread from $1 \cdot 10^{-3}$ to $1 \cdot 10^{-4}$. In addition, if stacking is to be used to increase the stored beam intensity, electron cooling is obligatory. The cooling time for a multi-turn injected beam varies with, among other things, the ion mass and charge, and ranges from $0.8 \mathrm{~s}$ for $\mathrm{A}=25$ to $0.2 \mathrm{~s}$ for $\mathrm{A}=200$ for typical electron cooling parameters. 


\subsection{Loading rate}

The repetition rate of the REX-ISOLDE post-accelerator is mainly determined by the breeding time inside the charge breeder which ranges from $20 \mathrm{~ms}$ for light ions (in this case limited by the cooling time in the Penning trap) to over $300 \mathrm{~ms}$ for neutron-rich heavy elements with $\mathrm{A}>200$. If REX-ISOLDE was to act as the injector for TSR, the operation mode has to be changed and the ions kept in the low-energy stage for a significant time, ideally up to several seconds. This is imposed by the electron cooling and the measurement time. Storage times of at least $1.5 \mathrm{~s}$ inside the REX-ISOLDE Penning trap has been demonstrated for $\mathrm{K}^{+}, \mathrm{Ni}^{+}$and $\mathrm{Rb}^{+}$ ions, although significantly shorter storage times are to be expected for noble gas ions. To maintain the transmission efficiency it is mandatory to keep the beam intensities from ISOLDE below $1 \cdot 10^{7}$ ions/s. A typical sequence for a nucleus with $\mathrm{Z}=50$ could be: ion collection from ISOLDE into the Penning trap for $1.5 \mathrm{~s}$; ion bunch transfer to the charge breeder and breeding for approximately $0.15 \mathrm{~s}$, post-acceleration and multi-turn injection into TSR, electron cooling for $0.35 \mathrm{~s}$, physics measurement for $1 \mathrm{~s}$ until the next injection occurs. The injection repetition rate would be $0.67 \mathrm{~Hz}$. The operation mode is suited for ions with half-lives above $1 \mathrm{~s}$.

\subsection{Multi-turn injection}

The pulse length of the accelerated REX-ISOLDE beam is given by the extraction time from the charge breeder, and is typically between 50 and $100 \mu$ s (FWHM). By applying a voltage ramp during extraction the pulse length can be decreased, most likely to less than $30 \mu \mathrm{s}$. Such short pulse length is essential to achieve an efficient multi-turn injection into the ring and not lose the exotic ions. An even shorter pulse length would result in a decreased electron cooling time and smaller beam radius for the non-cooled beam.

\subsection{Beam intensity limits}

It has been demonstrated that beam intensities up to $1 \mathrm{~mA}$, or somewhat higher for heavy highly charged ions, can be stored in TSR without beam instabilities caused by space-charge induced incoherent tune shift. The highest beam intensities extracted from REX-ISOLDE so far were $1.2 \cdot 10^{7}$ ions/s of ${ }^{10} \mathrm{Be}^{3+}$ and $3 \cdot 10^{7}$ ions/s of ${ }^{110} \mathrm{Sn}^{27+}$. Assuming a ring storage time of $5 \mathrm{~s}$ and a beam energy of $10 \mathrm{MeV} / \mathrm{u}$ that corresponds to $9 \mu \mathrm{A}$ and $200 \mu \mathrm{A}$, thus far below the stability limit.

\subsection{Internal target}

An internal gas target, with $\mathrm{H}_{2}$ or $\mathrm{He}$ as gas, is foreseen for different reaction studies. Ideally the gas target thickness should be variable from $5 \cdot 10^{11}$ to $5 \cdot 10^{13}$ atoms $/ \mathrm{cm}^{2}$, and the target extension in the beam direction be limited to $1 \mathrm{~mm}$. These requirements are challenging to achieve so different alternatives such as supersonic gas jet, cluster and pellet targets need to be investigated. 


\subsection{Storage life-time}

The life time of the radioactive ions in the ring is governed by the decay time and various loss processes, such as multiple and single Coulomb scattering, radiative and dielectronic recombination in the electron cooler, and electron capture and stripping by the residual or the target gas. The integrated thickness of the residual gas for a baked system is approximately $7 \cdot 10^{9}$ atoms $/ \mathrm{cm}^{2}$, thus significantly thinner than the internal target anticipated. When operating TSR in Heidelberg without an internal target storage, times ranging between several tens of seconds to even hours have been reported [4]. Although, with an internal target as specified above it turns out that the life times are reduced significantly due to electron capture and stripping processes in the target. If we assume an $\mathrm{H}_{2}$ target thickness of $1 \cdot 10^{13}$ atoms $/ \mathrm{cm}^{2}$ and a beam energy of $10 \mathrm{MeV} / \mathrm{u}$, the half-lives for the heaviest elements with charge states attainable by the present breeder will be at least $0.5 \mathrm{~s}$. For light elements the storage time is significantly longer. Likewise, if higher ion-charge states are assumed, either by an upgrade of the breeder or by making use of electron stripping after the Linac, the storage time can be increased by approximately a factor 10 .

\subsection{Beam cleaning}

By the use of beam scrapers inserted horizontally at a point in the ring where the $\beta$ function is small and the momentum dispersion large, unwanted beam contamination can be removed. The mass resolution is limited to around 4000 if TSR is operated in the normal mode. Thus the applicability for suppression of isobaric contamination from ISOLDE is limited, but stable contamination on a similar mass-to-charge ratio originating from the charge breeder can be removed in some cases. Mass selective acceleration is another method that could be explored for beam cleaning.

\subsection{Charge breeder upgrade}

The present charge breeder at REX-ISOLDE has limited performance in terms of charge states as it was only designed to produce mass-to-charge ratios less than 4.5. It has the capability of producing fully stripped ions up to Z 19 with some efficiency if the breeding time is increased. As it stands now some suggested experiments require fully stripped ions up to $\mathrm{Z} 60$ and few electron systems (Li- or Na-like) up the heaviest elements. To reach such high charge states an upgraded charge breeder is compulsory and it would have to be a state-of-theart SuperEBIT. Such an upgrade is not part of the first suggested phase for the TSR at ISOLDE project. Higher ion charge states would also be beneficial as the storage lifetime is increased in most cases, as mentioned above. In addition, to store neutron-rich ions with $\mathrm{Z}>70$ at $10 \mathrm{MeV} / \mathrm{u}$, a higher charge state than achievable with the present charge breeder is necessary from a ring rigidity point-of-view, but can be obtained by electron stripping at the end of the Linac.

\subsection{Storage ring positioning}

The existing experimental hall extension at ISOLDE is going to host part of the superconducting Linac for the beam energy upgrade and several experimental stations. The most logical positioning of TSR would be in a separate hall next to the hall extension (see 
Figure 1). Due to infrastructure constraints the ring has to be placed approximately $2.8 \mathrm{~m}$ above the HIE-ISOLDE experimental beam lines.

\section{Conclusions}

It has been shown that the operation of TSR as a storage ring for radioactive ions produced and accelerated by ISOLDE/REX is feasible. An internal gas target can also be used for reaction experiments if the target thickness is adjusted in order to obtain acceptable life-times. The machine combination would open up several novel possibilities for physics. Presently a detailed study of physics cases in reach and the technical aspects of the setup is being performed.

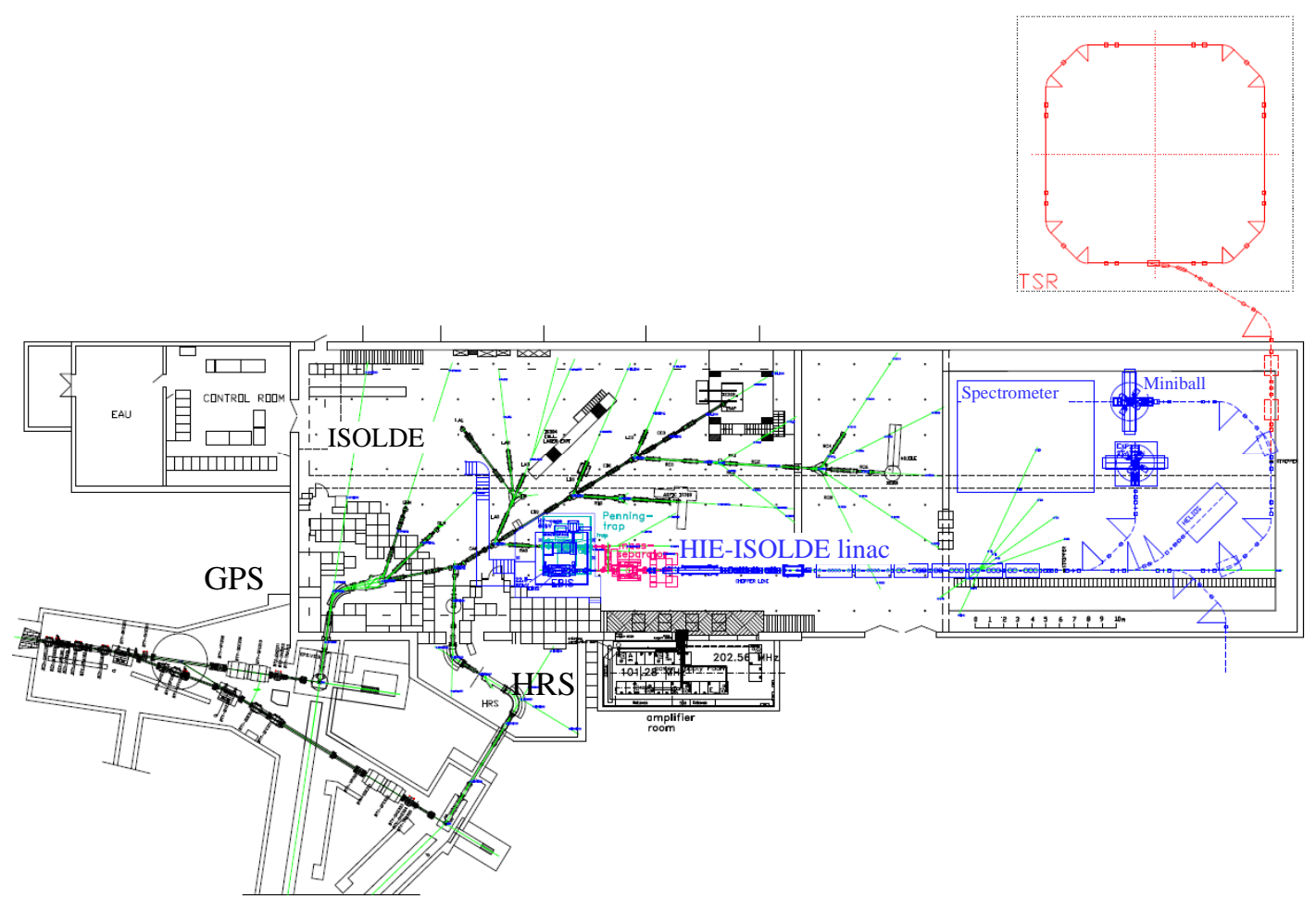

Figure 1. A drawing of the ISOLDE facility outlining the HIE-ISOLDE Linac and beam lines in blue and a possible placement of TSR in red.

\section{References}

[1] P. Baumann et al., The Heidelberg heavy ion test storage ring TSR, Nucl. Instrum, Meth. A268 (1988) 531-537.

[2] D. Habs et al., First experiments with the Heidelberg test storage ring TSR, Nucl. Instrum, Meth. B43 (1989) 390-410.

[3] Y. Litvinov ed., Technical Design Report, Storage ring facility at HIE-ISOLDE, to be submitted to CERN INTC committee Jan 2012. 
[4] O. Kester et al., The REX-ISOLDE project, Hyperfine Interactions 129 no.1-4 (2000) 43-66.

[5] M. Pasini et al., A SC upgrade for the REX-ISOLDE accelerator at CERN, Proc. Linac'08, Victora, Canada Sept 2008. 